\title{
CONVECTIVE OVERSHOOTING AND THE OBSERVED WIDTH OF THE MAIN-SEQUENCE BAND
}

\author{
R. NAPIWOTZKI, A. RIESCHICK, T. BLÖCKER, \\ D. SCHÖNBERNER, AND V. WENSKE \\ Institut für theoretische Physik und Sternwarte der Universität Kiel \\ Olshausenstr. 40, W-2300 Kiel, Germany
}

For many years we are witnessing a lively debate on the existence and extent of convective overshooting, mainly in the cores of main-sequence stars. This is an important issue, since even a small amount of overshooting increases considerably the mass of the finally hydrogen exhausted core and lenghthens the main-sequence lifetime correspondingly. The available evolutionary calculations assume either moderate overshooting, $d / H \mathrm{p}=0.25,(d=$ overshooting distance, $H_{\mathrm{P}}=$ pressure scale height; Maeder \& Meynet 1988) or strong overshooting, $d / H_{\mathrm{p}} \approx 0.50$ (Bertelli et al. 1986). Presently theory is unable to quantify the exact amount of overshooting, and one has to resort to empirical determinations.

Recently, Stothers (1991) collected all available information from the literature on stellar parameters and evolutionary calculations and concluded that, within the errors, $d / H_{\mathrm{p}}=0$ is an acceptable result, with a conservative upper limit of $d / H_{\mathrm{p}}<0.2$. However, such an approach is hampered by observational errors (like distance or temperature uncertainties, rotation) that are difficult to quantify and that may mask any definitive result. Detailed investigations of detached binaries may help in this matter (Andersen et al. 1990) but the number of suitable binary systems is probably not very large.

Recently, Napiwotzki et al. (1991) proposed a new method, viz. the direct determination of surface acceleration $g$ and effective temperature $T_{\text {eff }}$ by means of model atmospheres and comparison with evolutionary calculations. This method is distance independent and allows a large number of stars to be investigated. It takes advantage of the fact that the stellar evolution is strongly accellerated after the end of the core hydrogen burning, leading to only a very small number of stars in the region above the TAMS (Terminal age main sequence). One needs, however, spectrograms of good quality. The first application of this method by Napiwotzki et al. indicated an overshoot distance intermediate between the values proposed by Maeder \& Meynet (1988) and Bertelli et al. (1986). It was noted before by Lemke (1989) and by Wolff (1990) that the observed width of the main sequence in the $g-T_{\text {eff }}$ diagram do not agree with predictions from standard theory.

We report here on the continuation of this method: we determined $g$ and $T_{\text {eff }}$ of 16 bright B- and A-type stars of the field and computed new evolutionary tracks with the most recent opacities (Iglesias \& Rogers 1991). 


\section{OBSERVATIONS AND FITTING PROCEDURE}

The observed B- and A-type filed stars were randomly selected from the list of photometric $\beta$ standard stars of Crawford \& Mander (1966) for calibration purposes (Napiwotzki et al. 1992). The stars were observed with the Coude spectrographs of the $2.2 \mathrm{~m}$ telescope at the Calar Alto Observatory, equipped with a RCA CCD, and of the MCDonald Observatory, Texas, USA, equipped with a TI CCD. The (reciprocal) dispersion ranges from $7 \AA / \mathrm{mm}$ to $17 \AA / \mathrm{mm}$, and the images were centered on $\mathrm{H}_{\gamma}$ or $\mathrm{H}_{\beta}$.

Effective temperatures were derived by using existing photometric data $(u v b y \beta)$ according to the prescriptions of Moon \& Dworetsky (1985). This temperature determination appears to be very accurate $(\approx \pm 150 \mathrm{~K})$ and agrees very well with that based on UV fluxes (Napiwotzki et al. 1992; Wenske \& Schönberner 1992).

With $T_{\text {eff }}$ fixed, surface accelerations and (projected) rotational velocities were derived by matching theoretical line profiles, computed with Kurucz's fully line blanketed model atmospheres, to the observed ones. A detailed list of results is given in Napiwotzki et al. (1992). The fit error of $\log g$ is in most cases smaller than 0.05 dex. Together with the temperature uncertainty, the total error of $\log g$ is not larger than only 0.1 dex!

\section{MODEL CALCULATIONS}

We calculated several grids of evolutionary stellar models in the mass range of 2.0 $M_{\odot}$ to $8.0 M_{\odot}$. To determine the influence of opacities on the width of the main-sequence band three different sets of opacity tables were used: Cox \& Stewart (1970; CS), Huebner et al. (1977; LA), and Iglesias \& Rogers (1991; LL). The software used for all calculation was a modified version of the Kippenhahn code described e.g. in Schönberner $(1979,1983)$. For all sequences the chemical mixture was adopted to be $(\mathrm{X}, \mathrm{Y}, \mathrm{Z})=(0.699,0.28,0.021)$. The mixing length parameter (ratio of convective mixing length to local pressure scale height) was set to 1.3. With the LL opacities model sequences for three different overshooting parameter were calculated $\left(d / H_{\mathrm{P}}=0.0,0.15,0.25\right)$.

\section{DISCUSSION}

In Fig. 1 we show the position of ZAMS and TAMS calculated with the LL opacities and different overshoot parameters $d / H_{\mathrm{p}}$ in the $g$ - $T_{\text {eff }}$ plane. Added are the positions of the observed field stars. It is obvious that the standard tracks calculated with $d / H_{\mathrm{p}}=0$ are still not sufficient to fit the observations. A best fit would be possible with $d / H_{\mathrm{p}} \approx 0.15$. A comparison with our consistent calculations using the older LA and CS opacities shows that the location of the TAMS is dependent of the used opacity tables: higher values of $d / H_{\mathrm{p}}$ are necessary to fit the observations. We want to emphasize that, though we used the most recent opacities of Iglesias and Rogers (1991), the comparison with empirical data shows that the observed width of the main-sequence band is only reproducable with $d / H_{\mathrm{p}}>0$. 


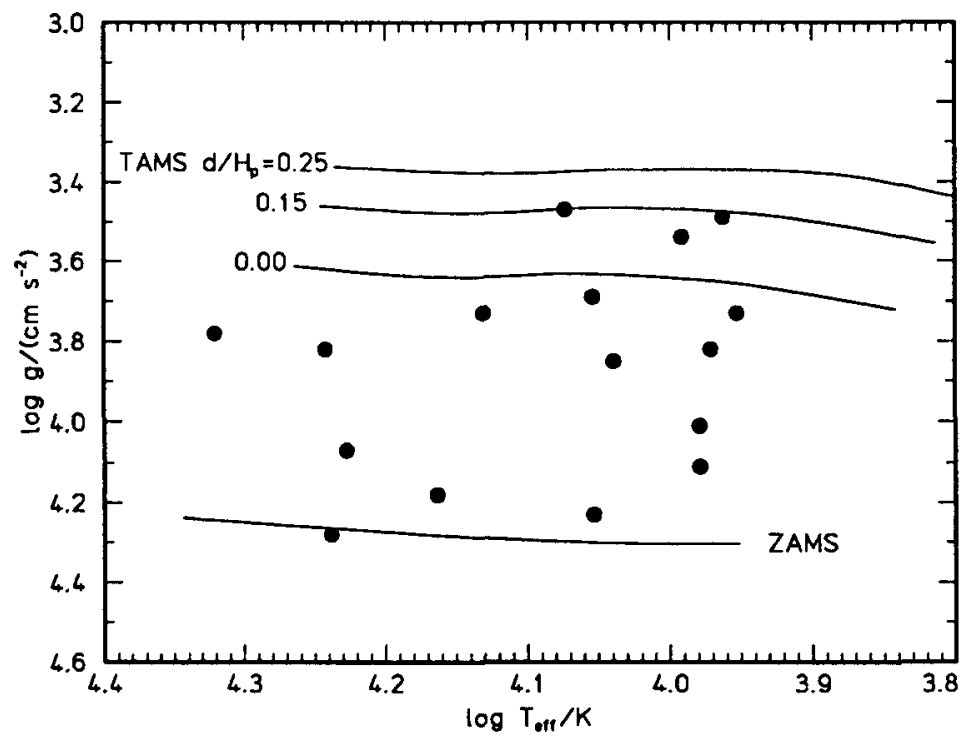

FIGURE I ZAMS and TAMS calculated with the LL opacities in the $\log T_{\text {eff- }} \log g$ diagram together with the positions of the observed stars.

\section{ACKNOWLEDGMENTS}

We like to thank Mounib El Eid for his support in installing the new opacity tables.

\section{REFERENCES}

Andersen, J., Nordström, B. Clausen, J.V., 1990, ApJ 363, L33

Bertelli, G., Bressan, A., Chiosi, C., Angerer, K., 1986, A\&AS 66, 191

Cox, A.N., Stewart, J.N., 1970, ApJS 19, 243

Crawford, D.L., Mander, J., 1966, $A J$ 71, 114

Huebner, W.F., Merts, A.L., Magee, N.H.Jr., Arog, M.F., 1977, Los Alamos Scientific Laboratory Rept., No. LA-670-M

Iglesias, C.A., Rogers, F.J., 1991, ApJ 371, L73

Lemke, M., 1989, $A \& A$ 225, 125

Maeder, A., Meynet, G., 1988, $A \mathcal{E} A S$ 76, 411

Moon, T.T., Dworetsky, M.M., 1985, MNRAS 217, 305

Napiwotzki, R., Schönberner, D., Weidemann, V., 1991, $A \& A$ 243, L5

Napiwotzki, R., Schönberner, D., Wenske, V., 1992, $A \& A$ in press

Schönberner, D., 1979, A\&A 79, 108

Schönberner, D., 1983, ApJ 272, 708

Stothers, R.B., 1991, ApJ 383, 820

Wenske, V., Schönberner, D., 1992, these proceedings

Wolff, S.C., 1990, $A J$ 100, 1994 